\author{
Italique \\ Italique \\ Poésie italienne de la Renaissance
}

XVII | 2014

Varia

\title{
«Benché 'l sol decline / vince un solraggio suo tutte le stelle». La parabola amorosa nelle Rime di Celio Magno
}

Giacomo Comiati

\section{(2) OpenEdition}

Journals

Edizione digitale

URL: http://journals.openedition.org/italique/388

DOI: $10.4000 /$ italique.388

ISSN: 1663-4438

Editore

Librairie Droz

\section{Edizione cartacea}

Data di pubblicazione: 1 ottobre 2014

Paginazione: 103-140

ISBN: 978-2-600-01841-8

ISSN: 1423-3983

Notizia bibliografica digitale

Giacomo Comiati, « «Benché 'I sol decline / vince un solraggio suo tutte le stelle». La parabola amorosa nelle Rime di Celio Magno », Italique [Online], XVII | 2014, online dal 01 octobre 2016, consultato il 21 avril 2019. URL : http://journals.openedition.org/italique/388 ; DOI : 10.4000/ italique.388 


\title{
G I A C O M O C O M I T I
}

\author{
«B E NCH È 'L SOL DECLINE \\ V I N C E U N S O R A G G I O \\ S U O T U T T E LE S T E L L E ». \\ LA PARABOLA A M OROSA
}

NELLE RIME DI CELIO MAGNO 



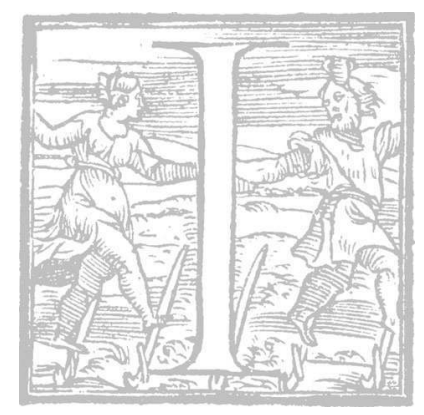

I canzoniere di Celio Magno (I536-I602) venne pubblicato a Venezia per $i$ tipi di Andrea Muschio nel I600 in un'edizione autorizzata dall'autore, che raccolse insieme alle sue rime anche quelle dell'amico Orsatto Giustinian (I538I603) ${ }^{\mathrm{I}}$ Sebbene la stampa apparve solo due anni prima della morte di Magno, diverse composizioni ebbero una precedente ed autonoma vita editoriale, che, grazie ad una parallela circolazione manoscritta di molti altri suoi testi, procurarono al poeta la stima e l'ammirazione dei contemporanei. ${ }^{2}$

Il libro di rime si articola in 137 componimenti e presenta un'unica partizione interna di marca autoriale, quella che separa la silloge conclusiva di argomento religioso dal resto della raccolta (prima del sonetto I25, infatti, si incontra la titolatura «Spirituali»). Sebbene l'autore non suddivida con altre didascalie la restante materia poetica, il monolitismo di questa prima macrosequenza si dissolve nel momento in cui la raccolta viene osservata con più attenzione. Essa infatti appare strutturarsi in una serie di sezioni giustapposte, tematicamente molto coese al loro interno, ${ }^{3}$ che «si susseguono secondo un ritmo che fa pensare [...] alla successione dei petrarcheschi Trionfi». Infatti di «triumphorum fragmenta»s ha parlato la critica per definire il liber del poeta veneziano, sebbene in esso si possano ritrovare le tracce di un percorso esistenziale che l'autore compie allontanandosi progressivamente dal mondo e dalle sue passioni per giungere alla contemplazione della trascendente maestà divina. ${ }^{6}$ L'impressione che la raccolta, però, sia unicamente «popolata dalle opposte forze che si contendono il dominio dell'esistenza》 ha fatto definire il liber di Magno un "canzoniere senza «Laura» [...] in cui le diverse liriche tendono [...] a convergere intorno ad una pluralità di centri». ${ }^{8}$

Uno di questi centri (e numericamente il più preponderante: infatti circa $i$ due terzi delle liriche della raccolta declinano questa tematica) è quello costituito dalle rime di argomento amoroso. Come si cercherà di dimostrare però, esso non è collocato casualmente all'interno del canzoniere, ma occupa una precisa posizione nell' architettura compositiva del liber, fortemente legata alle sillogi che lo precedono (rime 27-42, di tonalità funebre) e che lo seguono (rime I25-I37, di argomento spirituale). 
Spesso la critica ha posto l'accento su altri aspetti della produqione di Magno (focalizzandosi, ad esempio, sul tema della morte che attraversa l'intero suo canzoniere o sulle composizioni spirituali che lo chiudono), tralasciando l'ampia serie di componimenti d'amore che rappresenta, invece, un elemento centrale della raccolta. Anche se, forse, uno dei tratti più interessanti del poeta veneziano è rappresentato proprio dal fatto che egli non tratta unicamente la materia amorosa nel suo liber, ciò non implica che quest'ultima non debba meritare un'attenzione particolare, soprattutto se, analizzandola, essa non appare semplicemente una pedissequa rimodulazione dell'iter su cui sono costruiti molti canzonieri rinascimentali, ma è capace di vivificarne gli aspetti più topici tramite gli spunti offerti dalla rilettura dei classici (soprattutto di Orazio e di Properzio) e tramite l'inserimento di specifiche istanze di carattere autobiografico. Inoltre, per mezzo dell'articolato percorso esistenziale che è tracciato all'interno di questa sezione sentimentale, il poeta offre all'intera raccolta quel movimento interno, cui si accennava, che prima gli permette di cantare $i$ suoi amori terreni e poi lo invita a meditare sulla loro precarietà $e$ ad allontanarsene per contemplare l'infinita grandezza divina.

Sulla base di quanto affermato, questo lavoro si propone di analizzare la parabola amorosa che viene delineata da Magno nel suo libro di rime, parabola che si sviluppa nell'arco di circa settanta componimenti (dal sonetto 46 al sonetto I24, anche se la serie è interrotta da una sezione di tematica celebrativa - rime 99-109) e che, in accordo con le diverse fasi della storia d'amore di Magno con la sua amata, può essere scandita, come si cercherà di dimostrare, in cinque sillogi di componimenti (46-54, 55-69, 70-86, 87-98 e II0-I24).

Ma prima di porre l'attenzione su queste ultime, è opportuno soffermarsi per qualche istante su tre liriche della raccolta (43-45) che si frappongono tra la prima serie di argomento amoroso e la sezione che la precede, di tonalità funebre (27-42). Questi tre componimenti fungono da "cuscinetto" tra le due sillogi e offrono una concreta testimonianza della calibrata abilità costruttiva di Magno, che non giustappone semplicemente le diverse sezioni nella sua raccolta, ma, al contrario, ne articola l'alternanza in accordo con una precisa architettura compositiva.

La prima di queste composizioni di "cesura" è la canzone 43, Vago augellin gradito, che segna un importante scarto all'interno del canzoniere. In forte antitesi rispetto alla lirica che la precede (che chiu- 
deva, in tono disperato, la sezione funebre), essa declina il tema del locus amoenus, già molto caro a Magno (cfr. son. s e б), facendogli, però, qui assumere $i$ tratti salvifici dell'unico riparo in cui l'nomo possa sfuggire alle sofferenze del vivere derivate dalla meditazione sull'irreversibile caducità dell'esistenza." Manifestandosi quasi "per necessità" al fine di porre un freno alla disperazione soggiacente alla serie funebre appena conclusa, la canzone consente una netta svolta all'interno del percorso disegnato nelle rime: il paesaggio idillico diviene uno schermo, concepito e modulato in accordo con il modello oraziano, contro le inquietudini che derivavano dalla severità e dall'irrisolvibile tensione filosofica della sezione precedente e consente una nuova rinascita del canto poetico. Si noti che è solo a partire da questo momento che nel canzoniere il poeta declinerà la tematica amorosa. ${ }^{\text {. }}$

In questa prospettiva la canzone 43 e quella che la precede, Piangea l'acerbo fin Tirsi dolente, assumono una grande importanza nell'architettura delle rime di Magno. I due componimenti giustapposti rappresentano uno il termine della sezione mortuaria e l'altro l'apertura della successiva, secondo uno schema già presente nelle Rime di Giovanni Della Casa, che, come si cercherà di dimostrare, costituisce un importante modello per il poeta veneziano. " La canzone 43, dopo un'apostrofe al «vago augellin» dalle tonalità petrarchesche ( $v v . I-I 3) e$ dopo aver descritto il locus amoenus dove l'animale vive ( $v v$. I4-32), dà voce al sentimento del poeta che vorrebbe avere come l'animale le ali per «girne a volo» (v. 54).

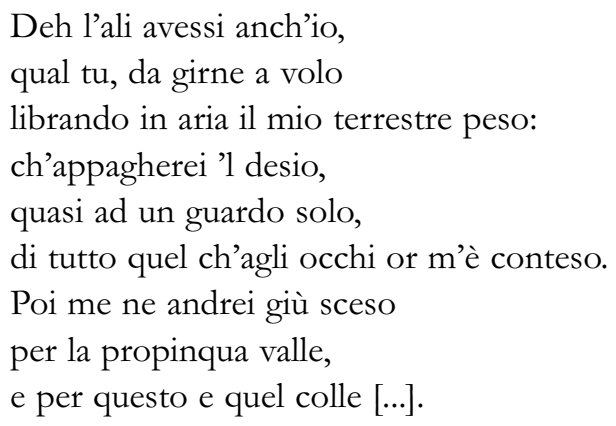

Alcuni banno sottolineato la vicinanza di questa canzone con luoghi petrarcheschi e bembiani, ${ }^{\mathrm{I}}$ nonché la sua affinità ad alcuni noti passi leopardiani, ${ }^{\mathrm{I}}$ ma $i$ versi sembrano alludere in modo puntuale anche ad 
un altro illustre precedente, molto vicino alle corde del poeta. Si fa riferimento a Giovanni Della Casa, ed in particolare al suo sonetto 63 Già lessi, et hor conosco in me, sì come. In esso Della Casa sviluppa il tema dell'anima che, appesantita dal corpo, non può elevarsi verso il cielo. Si leggano le terzine del componimento:

Lasso: e soviemmi d'Esaco, che l'ali d'amoroso pallor segnate ancora digiuno per lo cielo apre e distende, e poi satollo indarno a volar prende: sì 'l core anch'io, che per sé leve fora, gravato ho di terrene esche mortali. ${ }^{14}$

$(9-14)$

Esaco, trasformato in cormorano, se è digiuno (quindi senza il peso della corporeità), può aprire l'ali «per lo cielo», mentre se è «satollo», «indarno a volar prende». ${ }^{15}$ Immediate sono le corrispondenze che si possono tessere con la stanza di Magno dove il poeta, pesante del suo «terrestre peso», invidia l'«augellin» che può volare libero, sebbene le motivazioni in accordo con le quali $i$ due autori declinano questo tema differiscano non poco: a due liriche di distanza dalla conclusione del suo canzoniere, il fiorentino sviluppa questo motivo come memento per invitare l'nomo a liberarsi «di quanto di falso e di eccessivo lo intralcia e lo appesantisce», ${ }^{16}$ mentre Magno, nell'invidiare il volo dell'animale dà voce, ad una "platonica ansia di fuga dal mondo, sognando ali per la propria anima». ${ }^{17}$ Quest'ansia si potrebbe leggere come il frutto della rifrazione dell'onda poetica che ha dato vita alla lirica: essa non contraddice la tranquillità della pace offerta dal locus amoenus, ma può essere, invece, interpretata come il prosieguo inerziale del movimento di fuga dalla sofferenza intrapreso nella canzone come rimedio all'inquietudine scaturita dalla meditazione declinata nella sezione funebre appena conclusa.

Il secondo componimento della "cerniera" tra la sezione amorosa e quella che la precede è il sonetto 44, Cangi or beato il Po, cangi il Metauro, scritto per le nozze di Lucrezia d'Este con Francesco Maria, duca di Urbino. L'autore riproduce qui per analogia lo schema adottato all'inizio della silloge mortuaria, in cui una composizione di tono encomiastico in ricordo di una nobildonna, Margherita di Francia (son. 30, Mira dal ciel ove beata or vivi) apriva - dopo le due 
canzoni in morte di Irene di Spilimbergo e di Elena Mazza - la serie dei sonetti. In questo caso, dopo la canzone Vago augellin gradito, il tema amoroso è introdotto per mezzo di un epitalamio rivolto alla marchesa d'Este.

La terza lirica della "cornice", la 45, varia la medesima tematica, utiliz:ando un'immagine del mito classico: quella di Giove che avanza incatenato davanti al carro di Amore. ${ }^{\text {I }}$ Il sonetto può essere foriero di due significati: da una parte, sottolinea l'universalità della passione erotica, di fronte alla quale non solo gli dèi, ma anche il loro padre ̀̀ inerme, dall'altra - quasi come se fosse un'implicita excusatio non petita dell'autore - diviene lo strumento con cui il poeta cerca di giustificare velatamente il sentimento d'amore che dal componimento successivo confesserà di aver provato. A partire dalla lirica 46, infatti, viene propriamente declinata la parabola sentimentale.

A differenza di molti poeti cinquecenteschi (e dello stesso Petrarca), il Magno non comincia la trattazione della tematica amorosa a partire dall'innamoramento, ma - secondo l'illustre esempio di Della Casaapre la vicenda d'amore in medias res, presentandosi già innamorato di una donna crudele che non lo ricambia e che non crede nemmeno all'autenticità del suo sentimento. Il primo verso del sonetto 46, componendosi di una congiunzione causale ("poiché») seguita da due avverbi di negazione («né»), implicitamente allude alla presenza silenziosa di un discorso che era già in atto, nel mezzo del quale il lettore viene introdotto ex abrupto. Proprio con questo componimento, Poiché né il lungo mio gridar mercede, si può far iniziare la prima delle cinque serie di liriche in cui è possibile suddividere la parabola amorosa declinata dal poeta. La sezione si estende fino al sonetto 54 ed è caratterizzata da una forte unitarietà tematica, offerta dal motivo dell'amore sofferto.

Il sonetto 48, Mai non ritorno al mio bel sole amato, il terzo del primo ciclo, descrive la disperata situazione in cui il poeta si trova a vivere: quella di chi vorrebbe allontanarsi dalla donna amata poiché sa bene che standole vicino - dal momento che lei lo rigetta - la sua sofferenza non può che accrescere, ma, al contempo, non riesce a separarsi perché questo non può che recargli altrettanti tormenti, e quindi le torna accanto, venendo, però, puntualmente respinto. 
Mai non ritorno al mio bel sole amato seguendo i piè, ch'altrove andar non sanno: ché non sia del piacer maggior l'affanno, e da presso più acerbo il ben bramato.

Quegli occhi, i quai per adorar son nato, com'essi, ohimè, per sol mio strazio e danno, pur d'un guardo mercede al cor non danno, sempre in atto ver me nemico, irato.

E se lontan dal loro sdegno io vivo, più cruda guerra allor, misero, provo, del mio solo conforto e d'alma privo. Così vo del mio male ingordo e schivo, e quel che m'è più dolce, amaro trovo, disperato sperando, or morto, or vivo.

La contraddittorietà della sua situazione e la declinazione del topos del costante cambiamento di luogo come vano rimedio alle sofferenze dell'animo, seppur ben documentato nella tradizione letteraria, richiama in modo particolare il sonetto I4 di Della Casa, Cangiai con gran mio duol contrada et parte $e$, la quarta strofa della canzone 45, Amor, i' piango, et ben fu rio destino, del poeta fiorentino. ${ }^{19}$ I versi 52-5s di questa canzone ("Cosi corro a madonna, e neve e ghiaccio / le trovo il cor, e 'nvano / di quel nudrirmi, ond'io son si lontano, / col penser cerco; anzi più doglia abbraccio») costituiscono un significativo ipotesto per il sonetto di Magno, che sembra fonderli con un altro ricordo dellacasiano (Rime 5, 5-8, «Chéqualor torno al mio conforto, epresto / son, lasso, di nutrir l'alma digiuna, / trovo chi mi contrasta, e'l varco impruna / con troppo acerbe spine; ond'io m'arresto»).

Queste affinità riscontrabili con la poesia dellacasiana non solo sottolineano la vicinanza tra $i$ due poeti, ${ }^{20}$ ma soffondono anche di una luce sofferente e pensosa le prime liriche d'amore del veneziano. Nonostante la loro evoluzione futura sarà ben diversa da quella tratteggiata nelle rime del fiorentino, la vicinanza con queste ultime nella prima fase della raccolta permette a Magno di sfumare senza brusche interruzioni tonali il passaggio dal canto funebre appena declinato a quello amoroso. Il sonetto 52, Tra duo contrari, ognun per sé possente, segna un momento importante nella parabola che stiamo analizzando. Nella lirica si descrive la lotta che hanno intrapreso la ragione del poeta ed Amore. La prima vorrebbe spronare il poeta a vendicarsi dell'amata 
(variatio del tema del son. 50), mentre il dio «di scuse adombra il mal sofferto / e torna a ravvivar le fiamme spente» ( vv. 7-8). Interviene poi l'anima che, ergendosi a giudice della contesa, dichiara di voler seguire la proposta della ragione, ma Amore si oppone a questa risoluzione e con le sue arti «sforza e tira» (v. I3) il poeta finché egli non cede e si schiera dalla sua parte. La stessa esperienza era stata declinata anche da Della Casa nello spazio di due liriche: la I9, Sperando, Amor, da te salute in vano, e la 20, Ben foste voi per l'armi e 'l foco elette. Nella prima di esse il fiorentino si riprometteva di abbandonare l'amore, che gli aveva procurato null'altro che sofferenze, e di seguire un cammino di santità, ma nel sonetto successivo «la forza soverchiante della bellezza [della sua amata] giustificherà implicitamente il brusco abbandono del pentimento del componimento precedente e dei propositi li espressì. ${ }^{21}$ Parallelamente allo schema dellacasiano, ma scindendo $i$ momenti del percorso li descritto in modo diverso, Magno presenta nel sonetto 2 sia ilsuoproposito di abbandonare l'amore (ivv. 9-II lo indicano chiaramente: "Vinca lo sdegno e 'l suo velen m'inspiri / cosi adentro nel cor, ch'estinta giaccia / ogniindegna cagion de' miei sospiri»), sia la vittoria del dio contro la sua stessa volontà, mentre nel componimento seguente, il 53, Nero e crespo ha 'l bel crin madonna, e tale, rappresenta le bellezze irresistibili dell'amata che sono state le nuove armi con cui Eros ha reso vane le sueprecedenti intenzioni.

Proprio a partire dal sonetto 53 sembra di assistere ad una sorta di metamorfosi della donna: gli aspetti di «cruda guerriera» scompaiono o, almeno, si fanno irriconoscibili. Ma se si interpreta questa nuova veste dell'amata alla luce di quanto detto precedentemente, apparirà chiaro che non sono mutati $i$ suoi tratti, bensi il modo di percepirli da parte del poeta, che, arresosi ad Amore, non ba più lo sguardo velato dal dolore provocato dalle sofferenze e dal desiderio di vendicarsi di esse. La vittoria di Eros è stata tanto schiacciante (in questo Magno si allontana dal percorso del Della Casa) che da questo momento in poi il poeta si abbandona totalmente al dio e vive la sua vicenda amorosa in modo nuovo rispetto a quanto tratteggiato in precedenza. Tanto profondo è questo cambiamento che nel sonetto che segue nella raccolta, il 54, Mentre con aspre ingiurie in dolce canto, può addirittura trovare luogo (in antitesi rispetto all'immagine della «cruda guerriera» che sarebbe andata «altera» della morte del poeta) quella di una donna schiva che punge e riprende lo stesso Amore, invitandolo ad allontanarsi 
da lei. Eros, ferito dalla giovane che lo scaccia, per vendetta «fere con maggior forza ogn'uom che l'ode» (v. II), e, primo fra tutti, il poeta. Quest'ultimo, come conseguenza del gesto del dio, sembra amare con un sentimento diverso, come se fosse nato in lui un nuovo affetto. L'impressione è quella di essere di fronte ad un secondo inizio della storia d'amore, ad una sorta di "secondo innamoramento", segnalato forse anche dallinterruzione di una continuità di schema rimico che finoranelle quartine - non era mai venuta meno. Il sonetto 54, infatti, è l'unico in tutto il canzoniere di Magno a presentare le quartine a rima alternata (tutti gli altri le hanno incrociate) ed è sempre l'unico ad avere le terzine costruite secondo lo schema CDC EDE, non petrarchesco. La congiuntura di questi aspetti, assieme ad altri due esterni (ovvero la quasi totale assenza nei componimenti che seguono della tematica della sofferenza, che è presente, invece, insistentemente - come si è visto - dal sonetto 46 al sI, ed il fatto che da questo momento in poi l'autore canterà solamente le lodi della donna fino a raggiungere il coronamento della sua passione) suggeriscono che il componimento 54 possa essere considerato la lirica conclusiva di una sezione dedicata ai tormenti d'amore e, al contempo, il punto di avvio di un'altra sezione della parabola amorosa del poeta.

A partire dalla canzone 55, A che dagli occhi, Amor, vaghi e sereni, nel liber di Magno sembra aprirsi una nuova silloge che canta le gioie di un amore ricambiato (la seconda delle cinque cui si faceva riferimento, che si estenderà fino alla canzone 69). La presenza di una canzone nel momento preciso in cui l'autore decide di trattare un'altra tematica rispetto a quella che stava declinando in precedenza può lasciar supporre che questa forma metrica assuma nell' architettura compositiva del canzoniere una precisa funzione strutturale. Lo si poteva già ipotizzare una volta osservato che le canzoni 42, Piangea l'acerbo fin Tirsi dolente, e 43, Vago augellin gradito, erano collocate, la prima, a chiusura di una sezione di tonalità funebre e, la seconda, ad apertura del nuovo ciclo amoroso. La stessa funzione di spartiacque assumeranno (non casualmente) anche le canzoni 70, Dunque rea morte ha spente, 86 , Me stesso piango e de la propria morte, 87, Pur m'apri, o Febo, il desiato giorno, e 99, Pien di lagrime gli occhi e 'l cor di doglia. La 70 separa la silloge di amori felici che stiamo per analizzare dalla successiva, che canta gli affetti del poeta in absentia, essendo egli costretto a vivere per un lungo periodo di tempo 
lontano dall'amata. Come questa sessione caratterizzata dalla separazione dei due innamorati è chiusa dalla canzone 86, cosi la canzone 87 apre il nuovo ciclo di amori felici (rime 87-98), una volta che l'autore, tornato in patria, si ricongiunge con la sua donna. Quest'ultima serie, infine, è separata dalla successiva, in cui l'autore elogia la sua patria (componimenti 99-109), tramite l'inserimento della canzone 99, in morte di Domenico Venier. La tendenza che si è qui descritta e che si può ricondurre alla volontà autoriale di attribuire una funzione partitiva alla forma metrica della canzone non è disattesa negli altri contesti in cui viene utilizzato lo stesso metro, anzi, si può immaginare che l'architettura del canzoniere del Magno si costruisca proprio attorno alle Is canzoni presenti nel libro di rime che ne ritmano la struttura ed alternano le sezioni di cui si compone. ${ }^{22}$

Ma questo discorso ha una direttiva centrifuga rispetto al tema che ci siamo proposti di trattare in questo lavoro. Si ritorni ad osservare da vicino la parabola amorosa che dispiega l'autore nelle sue rime. Come si accennava in precedenza, la canzone ss apre una sezione di amori felici e segna l'approdo dei sentimenti del poeta a degli affetti più sereni (perché ricambiati).

Nella lirica ss l'autore si rivolge esclusivamente ad Amore, anche se, implicitamente, egli si indirizza all'amata, come ci suggerisce la didascalia posta nella «Tavola delle Rime» ("Cerca di persuadere la sua donna ad accettarlo per suo poeta»). Il poeta invita il dio a riflettere sul fatto che ben presto il Tempo lo scaccerà dal «bel ciglio» della sua donna, suo vero «regno» (v. I3). Per questo, dopo aver dipinto le possibili ingiurie che il nemico potrà causargli (vv. 27-33), ricorda ad Amore che mentre egli gli parla il Tempo «pur se n' vola, al suo danno passando. I Già gli par di vincitor mirarlo, / rotto a lui l'arco e spennacchiate l'ale, / e con doglia immortale / dal suo nido gentil tenerlo in bando» ( $v v$. 55-59). L'unico mezzo con cui si può contrastare la tirannia del nemico, aggiunge l'autore, è la poesia ( $N$ Non ba, se dritto miri, / più bel don da natura umana mente / od arte piu possente / a cosa oprar meravigliose e nove, / di quella che le muse al canto move», vv. 86-90). Di conseguenza, l'io lirico invita il dio a scegliersi un amico/difensore tra i poeti, cosi soltanto Eros potrà avere vittoria sul Tempo ("Di quei ch'a tal favor degnan le stelle / un solo scegli e tel procaccia amico; / ché del tempo nemico / ei sol darti potrà vittoria e palmà, vv. I09-II2). Poi prosegue: 
Ma pria che sovra alcun sentenza cada ch'a tanta impresa dar debba di piglio, apra la mente il ciglio, ed al deliberar spazio consenta.

[...]

Quanti ne sono al tuo pensier rammenta:

quei però che t'apriro i petti suoi

e che 'l guardo di tua donna infiamma:

ché chi non arde a l'amorosa fiamma

scema grazia cantando a’ pregi tuoi.

Colui s'elegga poi

ch'in amar primo ha più per te sofferto.

(I 27-I 30 e I 35-I 4I)

Magno con ogni evidenza, anche se implicitamente, desidera presentarsi come il miglior difensore che Amore possa scegliere. Il primo requisito che un poeta deve dimostrare di possedere per poter diventare il campione del dio è quello di aver sofferto per amore, tematica sviluppata, come si è visto, esattamente nel ciclo di sonetti che precede questa canzone. Proprio alla luce dei versi di quest'ultima, quella silloge sembra acquistare un secondo valore di carattere metaletterario, dal momento che testimonia le pene che l'autore ha già provate e che, dunque, ne provano l'eleggibilità a paladino di Amore.

La canzone ss si chiude, poi, con una preghiera rivolta al dio.

Ma non si tenga in ria prigione amara

qualunque avrai per sì bel vanto eletto,

né mercé lagrimando indarno chieda:

ch'ingegno in cui gran duol continuo fieda,

par che 'l canto e le rime aggia in dispetto;

e dal gravoso affetto

che respirar nol lascia, oppresso e stanco,

sul cominciar vien manco;

o se descrive pur suo duro scempio,

è di tua crudeltate indegno essempio.

Fa ch'anzi lieto ognor gridando ei chiami

te signor grato e sé felice amante;

e che d'aver si vante

quanto puote venir d'onesto dono.

Volgi pietoso in lui le luci sante, 
con cui da morte a vita altrui richiami;

rendi a lui dolci gli ami

ove i cor presi a tanto strazio sono.

Da quel saggio parlar cortese suono

e rinverdirla a più soave frutto:

talché sempre lontan da doglia e lutto

con l'ardor senta il refrigerio insieme.

E ciò fecondo seme

in lui sarà del tuo sperato onore:

ché dolcezza e stupore

versando in cantar lei, sua gran beltate

porterà viva ancor per ogni etate.

(I $53-179)$

Come ricompensa (e mez:.0) per il canto eternatore Magno chiede che chi sarà eletto campione del dio possa veder ricambiato il suo affetto da parte dell'amata. Si vedrà che nel prosieguo della raccolta tale preghiera verrà esaudita (e dalmomento che ne beneficeràproprio ilpoeta sipotrebbe immaginare post hoc che Amore abbia eletto lui come suo paladino).

In accordo con questa visione la canzone si pone in una posizione chiave all'interno della parabola amorosa raccontata dall'autore. Permette, infatti, di risemantizzare le liriche che la precedono, funzionalizzando le sofferenze li descritte in rapporto alle caratteristiche che il campione di Amore deve avere, ed al contempo, anticipando l'evoluzione della storia d'amore successiva, offre la possibilità di interpretare il coronamento di essa come l'esaudirsi della preghiera rivolta al dio nei versi finali della canzone, dando cosi forma coerente alla vicenda narrata in episodi separati. Il componimento, inoltre, assolve un'altra funzione di grande importanza. Anche all'interno di una parte del canzoniere prettamente rivolta al tema erotico Magno (con i toni più espliciti incontrati dall'inizio della raccolta) pone qui apertamente l'accento su uno dei temi cardine della sua poesia: il potere salvifico del canto poetico contro "l'aspra del tempo avida lima» (son. I, o). Che la trattazione di questo tema sia poi finalizzata ad obiettivi molto concreti sarà da considerare in modo relativo; molto interessante è la sua semplice presenza in questo luogo delle rime (come se l'autore - tramite un simile accenno - non volesse far dimenticare il tema con cui ha aperto il suo libro di liriche $)^{23}$. Il sonetto che segue nella raccolta, il numero 56, è in lode della donna amata. Dalla sua lettura si ricava unicamente l'impressione di un 
componimento costruito su loci rethorici tradizionali, collegati insieme tramite la risemantizzazione a fini elogiativi della tematica dantesca dell'insufficienza della parola umana nell'esprimere concetti troppo elevati. Ma esiste un'ulteriore lode celata tra le parole del sonetto. Secondo un gusto diffuso nella cultura rinascimentale, molto apprezzati tra $i$ componimenti encomiastici erano gli acrostici (detti anche liriche per li capiversi). Questa lirica, com'è segnalato dalla didascalia posta nella "Tavola delle Rime», è un sonetto per li capiversi: le prime lettere del componimento, infatti, formano le parole PAVLINA VITA MLA, che ci danno un'indicazione del possibile nome dell'amata del poeta (cui finora non si era fatto riferimento esplicito e che, anche in seguito, verrà celato dietro apostrofi di forte ascenden za classica: «Cinzia» nella lirica 62 e «Filli》, o «Fillide», nei sonetti 88-93). ${ }^{24}$

I due sonetti successivi (57, O begli occhi, de l'alma esca felice, $e$ 58, Pria risplender le stelle a mezzo il giorno) proseguono la serie dei componimenti elogiativi dalla donna. Nel primo il poeta canta la bellezza dei suoi occhi secondo la topica tradizionale, mentre nel s 8 descrive la potenza del sentimento che lo arde tramite lo schema dell'adynaton. La lirica seguente, la 59, Scrivi - mi disse Amor - ch'al mondo vive, tesse un ulteriore elogio della donna, ma sembra assumere anche un'altra funzione in nome di richiami interni alla raccolta. ${ }^{25}$ Il componimento potrebbe ricollegarsi implicitamente alla canzone 55 , come se in esso si realizzasse la prima delle due richieste che il poeta aveva rivolto ad Amore ("Di quei ch'a tal favor degnan le stelle / un solo scegli e tel procaccia amico», vv. I09-IIO). L'imperativo del dio che apre il sonetto 59 ("Scrivi-mi disse Amor), che richiama il precedente petrarchesco di Rvf 93, Più volte Amor m'avea già detto: Scrivi) potrebbe, infatti, far presumere che il poeta sia stato eletto come suo campione e debba ora elogiare la donna. A questo sonetto ed al seguente ci si riferiva quando si faceva accenno alle corrispondenze che si potevano riscontrare tra la canzone ss e $i$ componimenti successivi.

Nella lirica 60, in parallelo a quanto avviene nella 59, sembra che Amore esaudisca la seconda preghiera del poeta: «Ma non si tenga in ria prigione amara / qualunque avrai per si bel vanto eletto, / né mercé lagrimando indarno chieda》 (55, vv. I53-I5s). In questo sonetto, infatti, l'amata invita l'innamorato ad avvicinarsi a lei, mentre è seduta all'ombra di un parasole con un amorino che le regge uno specchio. ${ }^{26}$ Questa azione segnala un mutamento radicale nell' atteggiamento della 
donna: essa non si dimostra più sdegnosa, ma appare benevola verso l'amante.

L'importanza e l'eccezionalità di questi due componimenti sono segnalate anche per mezzo di una caratteristica strutturale delle liriche: in questi due sonetti lo schema alternato delle terzine (CDC DCD), utilizzato per oltre $i$ quattro quinti dei sonetti che compongono il canzoniere, è sostituito con quello non petrarchesco CDE ECD. ${ }^{27} \mathrm{La}$ sostanziale omogeneità della struttura rimica delle composizioni del canzoniere permette (e consente) di considerare particolarmente significativi quei tesi, come questo, in cui essa viene disattesa.

La sezione prosegue declinando dei temi quasi topici nei canzonieri rinascimentali: $i$ componimenti 63 e 64 trattano dei doni offerti dall' amata al suo innamorato, mentre il bs descrive una malattia della donna e la preghiera del poeta che ne chiede la guarigione. Nei versi finali di quest'ultimo sonetto si trova un'immagine particolarmente interessante. Il destinatario della lirica è Apollo, cui i due innamorati promettono, se egli risanerà l'amata, di onorarlo con infinite lodi e di ringraziarlo tante volte quanti saranno $i$ baci che Magno darà alla sua donna.

Sì canterem dapoi con puro zelo

te nobil padre del Castalio fiume, figlio di Giove e gran signor di Delo.

E quanti io darò baci al lor bel lume, che saran più che non ha stelle il cielo, renderò grazie al tuo cortese nume.

I versi delle terzine tramite l'uso della prima persona plurale nel verbo «canterem» (v. 9) alludono ad un equilibrio ormai ben stabilito tra $i$ due innamorati, mentre l'allusione al topos catulliano dei baci innumerevoli (cfr. Cat. s) lascia presagire che il loro legame amoroso verrà presto declinato in accordo con una maggiore componente sensoriale. Il primo luogo in cui questa si manifesta è il sonetto 67, Di notte in braccio al mio tesor godea, che celebra il coronamento della passione del poeta. In esso, infatti, viene cantata la prima notte d'amore con l'amata. Dopo i primi versi, però, la declinazione del tema sfuma velocemente ed assume $i$ contorni di un'ode al carpe diem. L'autore racconta di aver portato con sé un piccolo orologio per lasciare la casa della donna prima dell'alba, ma Amore ne rompe il meccanismo 
e fa si che la notte d'amore non venga interrotta e che il tempo che $i$ due innamorati trascorrono insieme si prolunghi fino al mattino. Le terzine sono occupate dal discorso diretto del dio che, confessato l'inganno, afferma di averlo fatto per insegnare ai due amanti a godere di ogni attimo di gioia, unico modo per vendicarsi del «tempo crudel» $\left(v . I_{4}\right){ }^{28}$ Se si ricollega questo sonetto alla canzone 55, sembra che Amore esaudisca qui pienamente la seconda preghiera del poeta ("Ma non si tenga in ria prigione amara / qualunque avrai per si bel vanto eletto, / né mercé lagrimando indarno chieda), 55, vv. I53-I55), che nella lirica 59 era già stata in parte assecondata. Dopo il coronamento della passione amorosa (qui esplicitato) il poeta puì ufficialmente chiamare Eros «signor grato»e sé stesso «felice amante» (come preannunciato al v. I64 della canzone 55$)$.

Ponendosi nel prolungamento tematico del componimento 67, il sonetto 68 descrive il primo incontro dei due innamorati dopo la notte trascorsa insieme. Si noti che la lirica occupa la posizione centrale nel canzoniere, circostanza che sottolinea la centralità del tema qui trattato. L'amata, felice di riabbracciare il poeta, lo bacia con affetto, mentre egli è rapito dai suoi dolci sguardi. Sebbene l'immagine possa avere un precedente nel sonetto I3 delle Rime di Ariosto, la matrice classica che vi soggiace è properziana. Nei versi finali dell'elegia II Is, infatti, si legge: «Tu modo, dum lucet, fructum ne desere vitae. I omnia si dederis oscula, pauca dabis» (vv. 49-50). Interessante è notare che questi sono i versi conclusivi dell'elegia $\mathrm{O}$ me felicem. o nox mihi candida, in cui Properzio celebra il primo incontro notturno con Cinzia. ${ }^{29}$ Sebbene Magno scinda in due componimenti distinti (rime 67 e 68) i momenti della celebrazione del coronamento della passione e quello in cui l'amata, riabbracciando il poeta dopo la notte d'amore, gli offre $i$ suoi baci, momenti che l'autore latino poneva in un'unica elegia, il poeta veneziano, per mezzo del ricorso allo stesso ipotesto, lega $i$ due testi in un elegante continuum narrativo, offrendo ai due sonetti un legame più stretto di quello che concederebbe loro la semplice affinità tematica.

Chiude questa sezione di "amori felici" la canzone 69, Quanto in voi, donna, io miro, a specchio rispetto alla canzone 5s, che l'aveva aperta. In essa il poeta canta la bellezza degli occhi dell'amata. La tematica collega il componimento al sonetto precedente (che si chiudeva con l'immagine degli «occhi beati»), ma, soprattutto, alla lirica 57 che aveva aperto la serie di rime in lode della donna. ${ }^{30}$ Si può forse notare, 
come ulteriore prova del fatto che la canzone chiuda la silloge, la presenza di un'immagine dalla forte rilevanza icastica alla fine del congedo: "Dunque, s'altro non posso, idoli miei, / porgovi [...] / 'l cor per voto» (vv. I03-I05).

A partire dalla lirica $7 I$, Poiché da rio destino altrove spinta, si assiste all'allontanamento del poeta dall'amata, «dovendo egli fare un lungo viaggio» (come si legge nella didascalia al sonetto presente nella "Tavola delle Rime»). Si scoprirà poi, grazie ai riferimenti espliciti offerti dalla canzone 86, l'ultima lirica del nuovo ciclo che qui si apre, che l'autore si riferisce al periodo della sua vita in cui visse in Spagna (dove rimase fino al 1578 ) al seguito dell'ambasciatore Andrea Biagio Badoer. ${ }^{31}$ Dal momento che tutti $i$ componimenti che vanno dal sonetto 7 I alla canzone 86 sono accomunati dal fatto che il poeta canta le sue sofferenze d'amore poiché si trova distante dall'amata, si puì individuare in questa sezione il terzo momento della parabola amorosa declinata dal poeta, dedicata agli affetti in absentia dell'autore, dopo la prima serie di amori sofferenti e la seconda di affetti corrisposti.

Tra questo nuovo ciclo ed il precedente decide di porre una canzone che assume anche in questo caso, come si ̀̀ già osservato, una funzione partitiva all'interno del liber. La canzone 70, Dunque rea morte ha spente, rappresenta un forte elemento di discontinuità con la materia trattata nelle sezioni ad essa adiacenti perché è un componimento in morte, dedicato alla memoria di Girolamo Molino, amico di Magno e, come ricorda il critico Antonio Pilot, "uno dei tre grandi sacerdoti del Bembo». ${ }^{32}$ La possibilità di inserire una canzone funebre all'interno di una serie pressoché ininterrotta di liriche amorose è già ammessa da Petrarca, ma Magno può trovarne esempio anche nelle Rime di Della Casa. ${ }^{33}$ Inoltre, dal momento che l'autore celebra Molino, «di libertà fervido amante» (v.29), uomo costante nel «ben oprar» (v.30) ed armato di virtù (v. 3I), come suo maestro morale (ed allude ad esso anche come sua guida poetica),${ }^{34}$ la canzone non rappresenta unicamente un momento di interruzione del discorso amoroso che il poeta sta declinando, ma assume anche un valore importante nel disegno autobiografico che percorre l'intero canzoniere. L'autore, infatti, rappresenta un molteplicità di affetti che non si esauriscono nei sentimenti suscitati dalla sua amata, ma abbracciano una pluralità di situazioni e figure.

Dopo la canzone 70, il poeta declina una gamma di situazioni il cui sottotesto è riconducibile alla sofferenza che egli prova per essersi allon- 
tanato dalla sua donna. I componimenti trattano del ritratto che il poeta lascia all'amata come pegno del suo affetto (son. 7I-73), della sua gelosia (son. 74-75), dei doni che le invia durante la sua assenza (son. 76), della sua rabbia per non aver ricevuto alcuna lettera da parte della donna e del suo rinnovato affetto dopo aver scoperto che ella gli aveva scritto, ma che i suoi messaggi erano stati bruciati insieme a molti altri perché provenienti da una città colpita dalla peste (son. 78-80). Segue nella sezione un breve ciclo, composto da cinque sonetti (8I-85), dedicato al tema (già petrarchesco e molto comune nelle raccolte cinquecentesche di poesia $)^{35}$ del tradimento dell'unus amor da parte del poeta, che pecca, invaghendosi di un'altra donna. Nella lirica 8I l'autore confessa questo secondo innamoramento, salvo comprendere subito il suo errore e pentirsene nel breve spazio dello stesso sonetto. Le quattro rime successive declinano un dialogo amebeo tra l'amante e il suo cuore sul medesimo tema. Lo schema è già presente in Petrarca (Rvf 242) e torna più articolato in Bembo (Rime 8 ) e in Della Casa (Rime 40), ma non esce mai dai confini del singolo componimento. Magno, invece, estende l'ampiezza delle singole battute del dialogo tanto da far occupare ad ognuna una lirica a sé stante. Sono proprio le didascalie a testo («Amante», riportata prima dei sonetti 82 ed 84, e «Cuore», prima delle rime 83 e 85) che avvalorano la possibilità di leggere la serie come $i$ vari momenti di un'unica conversazione.

La silloge dedicata agli affetti in absentia si conclude, infine, con la canzone 86, Me stesso piango, e de la propria morte, composta in Spagna nel I576, quanto il poeta temette di dover morire, come specifica la didascalia nella «Tavola delle Rime».36 Il componimento per metro e tematica risponde perfettamente ad un principio di bilanciamento rispetto alla canzone 70 che apriva la sezione, ma, al contempo, dà sfogo ad un sentimento autentico, che puì essere ricondotto a quell'ansia filosofica (orazianamente mai del tutto sopita), derivata dalla meditazione sull'ineludibile destino di caducità della vita umana.

A specchio rispetto a questo componimento si pone la canzone 87, Pur m'apri, o Febo, il desiato giorno, che apre la successiva serie di liriche (il quarto dei cinque momenti) in cui il poeta canta, una volta tornato in patria, l'affetto ritrovato della sua amata. Pur m'apri, o Febo, il desiato giorno è un componimento che si puì dire gemello di quello che lo precede, dal momento che entrambi presentano lo stesso schema metrico ( $A B b C B A a C C D E e D F F)$, anche se non ugual 
numero di stanze (nove in 86, cinque in 87). La diade, inoltre, costituisce, come le canzoni 42 e 43 o 69 e 70, un nodo nevralgico nell' articolazione delle sezioni del liber perché funge da spartiacque tra due momenti della parabola amorosa dall'autore. Il componimento acquista slancio dalla vena autobiografica della lirica precedente (che nella canzone 87 si dispiega come congedo preso dalla Spagna) e si struttura come un breve propempticon rivolto dall'autore a sé stesso prima del lungo viaggio di rientro a Venezia.

Una volta tornato nella sua terra natale, il poeta può rivedere la sua amata, cui si rivolge a partire dal sonetto 88, S'arde in me, cruda Filli, altro desio (come in tutta la sezione) con il nome di Filli, appellativo topico della poesia bucolica. ${ }^{37}$ Oltre all'onomastica, molti tratti di questa nuova sezione sono riferibili all'atmosfera arcadica, ma ciò sarà più evidente a partire dai successivi componimenti piuttosto che nel sonetto 88, il quale funge da cornice tra il nuovo ciclo di amori qui cantato e la precedente serie di componimenti composti in Spagna.

Nella lirica 88 si descrive il primo incontro dopo il lungo periodo di lontananza tra il poeta e l'amata, che si mostra ritrosa nei suoi confronti. Forse tale comportamento è imposto dalla topica, ma non è impossibile pensare che in esso la lirica possa pagare un tributo al ricordo poetico-biografico del tradimento ammesso in precedenza dall'amante (anche se il ricordo di tale atto si fa molto vago per mezzo della rarefazione dell'atmosfera bucolica del ciclo). La continuità con l'evoluzione narrativa interna ai componimenti che formano la raccolta non si manifesta solo nell'atteggiamento orgoglioso dell'amata, ma è allusa in modo esplicito nei versi iniziali del sonetto, dove si immagina che l'incontro tra $i$ due innamorati avvenga dopo una lungo periodo di separazione.

La scena idillica di ozio arcadico che viene descritta nel sonetto successivo, il numero 89, Qui sotto questo caro arbore amato, attesta il completo rappacificamento tra $i$ due innamorati. La donna è distesa all'ombra di un albero sui cui rami si è arrampicato il poeta, che le offre i frutti raccolti dalla pianta, immaginando quanta debba essere la loro gioia mentre piovono in grembo alla giovane (gioia che vorrebbe egli stesso provare tramutandosi in uno di quei frutti).

Qui sotto questo caro arbore amato

stava Fillide mia col grembo steso, 
mentr'io, su i rami a coglier pomi asceso, giù ne pioveva or d'uno or d'altro lato.

Gioir parea ciascun che per lei nato fosse, e da lei con festa accolto e preso; io de' più scelti ad arricchirla inteso nel suo goder per lor godea beato.

Quante fiate a' rai di quel bel volto intento e di me stesso in dolce oblio, fui quasi, errando il piè, sossopra volto!

E quante ancora indarno ebbi desio di cangiar forma, e 'n novo frutto volto caderle in sen tra gli altri pomi anch'io.

La scena arboricola, che sin dall'incipit si pone sulla scia dell'immaginario bucolico virgiliano, ${ }^{38}$ presenta dei tratti fortemente sensualistici, allusi tramite la variatio del topos classico della mela donata alla donna, richiamante un esplicito simbolismo erotico. ${ }^{39}$ Il poeta può permettersi di descrivere certe immagini in cui più vivo si presenta il sentimento passionale grazie allo schermo che gli viene offerto dall'ambientazione bucolica e dalla distanza che il mondo arcadico permette di interporre tra la realtà cantata e $i$ lettori (senza contare che alla poesia pastorale è statutariamente concessa la possibilità di riferirsi ad un certo repertorio amoroso che viene escluso dalla tradizione lirica petrarchesca). Ma si può ipotizzare che questa scelta poetica possa essere anche la conseguenza di una ponderata decisione stilistica antecedente. L'autore parla qui dei suoi amori dopo aver trascorso un lungo periodo di lontananza dalla sua patria e, di conseguenza, dalla sua donna. Il tono bucolico delle liriche potrebbe, allora, derivare anche dalla volontà di celebrare l'amata campagna veneta come luogo ideale, lontano da ogni turbamento, in cui (ancora una volta, orazianamente) il sentimento amoroso può vivere istanti di felicità nell'atemporalità del paesaggio arcadico.

Il sonetto 90, Perché con sì sottile acuto raggio, prosegue quello che potremmo definire "il ciclo di Filli". In esso l'ambientazione diurna finora incontrata cede ad una scena di amori notturni (molto simile a Rvf 237, 3I-36). Il poeta è steso con l'amata sotto un «frondoso antico faggio», quando la Luna, vagando alla ricerca del suo amato Endimione, li scorge e, scambiando erroneamente il poeta per il suo innamorato, impallidisce temendo di averlo trovato tra le braccia di un'altra. 
Nelle terzine il poeta le si rivolge, dicendole che il suo timore è vano perché mai Filli gli avrebbe preferito Endimione, né lui l'avrebbe mai potuta tradire nemmeno con Diana in persona.

Perché con sì sottile acuto raggio

Cinzia a spiar per l'ombra folta passi

dove Filli mia bella or meco stassi

sotto questo frondoso, antico faggio?

Forse, cercato il tuo pastor ch'oltraggio

ti fa tardo ver te movendo i passi,

qui gli occhi ancor per ritrovarlo abbassi,

e sospettosa in ciel fermi il viaggio?

Vano è 'l timor, se pur timor ti prese

in su 'l primo scoprir de' furti miei,

me credendo colui che 'l cor t'accese:

che per Endimion fuor del mio laccio

Filli non usciria; ned io torrei

Gioir Diana a te più tosto in braccio.

La lirica successiva, la 9I, Qual per bel prato a la stagion novella, $\grave{e}$ in lode di Filli, che viene elogiata variando un'immagine topica tassiana, quella dell'ape che punge le labbra della donna sulle quali si era posata credendo che fossero un fiore (immagine che viene evocata esplicitamente tramite il ricorso al v. 3 dell'espressione «ape ingegnosa», che si legge in entrambi $i$ luoghi tassiani in cui è descritto l'episodio: Aminta, I 443 e nel sonetto 89 delle rime per Lucrezia Bendidio). Magno, però, paragona l'ape che raccoglie il nettare dai vari fiori ad Amore, che prima distilla la dolcezza del volto e del seno dell'amata (assimilabili a dei fiori bianchi e vermighi) e che poi pone tale dolcezza sulle labbra della donna, baciando le quali si può godere di un nettare più prelibato di quello che viene servito agli dèi.

Qual per bel prato a la stagion novella

da vari fior va raccogliendo fuore ape ingegnosa il dolce, almo licore, di ch'empie e sparge la sua ricca cella; tal nel volto e nel sen de la mia bella Filli, or d'un bianco or d'un vermiglio fiore, coglie il dolce, cred'io, che serba in quella cara e soave bocca, industre Amore. 
Questo è 'l nettar d'Amor, ch'a mille prove vince quel che più dolce e più pregiato il bel frigio garzon ministra a Giove; e raro a me dal ciel gustarlo è dato: forse perch'uom mortal la via non trove da girne a par degli alti dèi beato.

La possibilità di godere delle grazie dell'amata congiunge il sonetto precedente a quello che lo segue nella raccolta, il 92, dove (con toni espliciti che richiamano quelli tassiani del sonetto Aminta, poi ch'a Filli non dispiacque ${ }^{40}$ viene descritta una scena d'amore passionale tra $i$ due innamorati. Il desiderio, spesso inappagabile, dell'amante trova qui soddisfazione e consente con una forzatura retorica ardita di tingere di connotati inusuali il topos del cuore del poeta che si ritira a vivere nel seno dell'amata

Con sue candide man del proprio petto Filli cortese il chiuso velo aperse, e duo piccioli colli a me scoperse tra cui valle giacea d'alto diletto.

Sculto per man famosa in marmo eletto sì vago seno il mondo unqua non scerse; né mai da sé formato agli occhi offerse Natura altro più vago e più perfetto.

Io, poich'a dolci prieghi in grazia l'ebbi, l'avide labbra d'alta sete pieno chinai, ma foco in quella neve bebbi.

E tutt'altro il mio cor pregiando meno ivi rimase, e mai più nol riebbi: ma felice or si vive in quel bel seno.

Lo schema alternato che si è prodotto nelle liriche precedenti (90-92) di componimenti in cui si cantano gli amori carnali dei due innamorati (son. 90 e 92) e di rime in lode di Filli (son. 9I) non viene disatteso dall'ultimo sonetto della serie, il 93, che tesse un nuovo elogio della donna. La scena bucolica dalle tonalità vespertine descrive la giovane mentre prende con la mano dell' acqua da un «cavo rame» ed irriga un giardino. L'inventio di Magno concede la parola ai fiori (come già nel sonetto 38 , Ecco di rose a questa tomba intorno), $i$ quali si dicono 
ricreati non già dall'acqua, ma dalla semplice vicinanza della candida mano di Filli.

Il componimento successivo, oltre che per il fatto di essere un'altra lode dell'amata, si lega al sonetto 93 grazie alla centralità che anche qui ha l'elemento floreale. Il poeta si rivolge alle «rose vermiglie» pregandole di non aprirsi al sorgere dell'aurora, ma di attendere il risveglio della sua donna, la sua «più bella Aurora». La lirica non è un sonetto (unico metro, insieme alla canzone, incontrato finora nel canzoniere di Magno), bensi un madrigale, ed è il primo di un breve ciclo di cinque (94-98). ${ }^{4 \mathrm{I}}$ L'impiego in questo momento della raccolta di una forma metrica medio-bassa e che è «per sua natura tutta volta agli amori> ${ }^{42}$ appare particolarmente affine al tono bucolico dell'atmosfera delle rime e dei loro protagonisti. ${ }^{43}$ Sebbene molto diversi siano gli schemi metrici delle cinque liriche (94-98) come anche i temi in esse trattati (dal paragone col mondo floreale nella 94, all'invito di "cogliere la rosa" nella 95, dalla gara canora tra un «augelletto» ed un pastore nella 96, alla traduzione di un epigramma dell'Antologia Planudea nella 97, ad un finto combattimento tra l'amata ed Amore nella 98), ${ }^{44}$ la serie trova nella poikilia un forte elemento di coesione interna, facendosi capace di tradurre in questa libertà di toni e di immagini la vitalità del mondo arcadico precedentemente descritto.

In perfetta simmetria rispetto alla canzone in morte di Girolamo Molino che precedeva la terza sezione amorosa del liber di Magno, la quarta è chiusa da un'altra canzone in morte, la numero 99, Pien di lagrime gli occhi e 'l cor di doglia, dedicata a Domenico Venier. L'improvviso riapparire della tematica funebre dopo una sezione arcadica fa pensare all'urgenza espressiva di voler affrontare il tema dell'onnipresenza della morte, che si insinua anche nel rassicurante mondo bucolico. La canzone, dunque, può essere intesa come una variazione del motivo classico Et in arcadia ego. Il poeta guarda con matura consapevolezza alla sorte inevitabile cui sono destinati gli esseri umani e si dimostra orazianamente cosciente di ciò anche quando cerca nell'angulus (declinato nella silloge appena conclusa nella sua componente di hortus conclusus) un possibile ristoro dagli altri mali che lo affliggono. Si può supporre che il manifestarsi della tematica mortuaria (che viene a concludere la serie bucolica) assuma $i$ tratti di un memento filosofico che il poeta rivolge a sé stesso oltre che al pubblico dei lettori.

Nella lirica 99 il poeta ricorda con affetto Domenico Venier, «il più grande petrarchista lagunare dopo il Bembo», ${ }^{45}$ e lo elogia sinceramente 
come suo amico fidato, come sua guida e come modello di virtù e di poesia. Ma Venier fu per Magno anche esempio di sincero amor patrio. Partendo da questo impulso, prende l'avvio nella raccolta una breve silloge di testi di ispirazione patriottica (I00-109) in cui l'autore esalta le glorie di Venezia e celebra la moralità dei suoi cittadini. La canzone 99, infatti, si lega ai sonetti che la seguono (I00-I06), condividendo con essi il tema encomiastico rivolto ad eminenti personaggi della Repubblica Veneta del tempo. Seguono, poi, due liriche (I07-108) di tono autobiografico (in cui il poeta, in accordo con le tonalità della serie, fa riferimento unicamente al suo patriottismo), ed infine chiude il ciclo, a specchio rispetto alla canzone 99, la 109, Ove, o Roma, son or l'altere imprese, in cui viene celebrata la gloria di Roma, uguagliata solo da quella della città lagunare. Nella silloge l'autore, deposti momentaneamente gli altri temi della raccolta (anche se, come si è visto, sono proprio questi che favoriscono la trattazione di quelli qui sviluppati), esalta la grandez?a di Venezia e gli nomini virtuosi che l'hanno resa e che la rendono tale.

Chiusa questa sezione, Magno delinea il quinto ed ultimo movimento in cui si articola la sua parabola amorosa (IIO-I24). La serie rappresenta un momento cardine nella raccolta, trovandosi in limine alla sezione spirituale (segnalata nel testo a stampa per volontà autoriale). ${ }^{46}$

La sezione assume, dunque, la funzione di cerniera tra le poesie religiose che la seguono e $i$ molti componimenti che la precedono, concretamente vincolati al mondo terreno. In accordo con tale posizione si comprende bene perché in questa sezione il poeta avverta la necessità di far evolvere ogni suo passato sentimento in uno più elevato, fino a raggiungere la disposizione d'animo appropriata per la meditazione religiosa e la definitiva celebrazione dei misteri cristiani e della trascendente maestà di Dio, cantati nell'ultima serie, e nei quali si può individuare il fine ultimo della tensione che attraversa l'intera raccolta. ${ }^{47}$ Per raggiungere quest'obiettivo appare indispensabile la rinuncia definitiva all' amore. Si può immaginare che in un autore come Magno, non solamente molto legato a questo sentimento, ma che è stato capace di presentarlo nei suoi più diversi aspetti e di cui ha cantato con sincerità anche le gioie, tale abbandono risulti essere non solo molto doloroso, ma anche particolarmente complesso da tratteggiare, non volendo contraddire quanto affermato in precedenza ed, al contempo, dimostrando di essere mosso a tale rinuncia da un autentico pentimento. 
Tale movimento che si è qui riassunto si può ritrovare già espresso in nuce nel primo dei quindici sonetti che costituiscono la sezione (il IIo, Mentr'ebbi in verde età fervido il sangue), il quale, da solo, sembrerebbe sufficiente a fare da cerniera per congiungere $i$ componimenti che lo precedono con la silloge finale delle rime spirituali. Ma la complessità del cambiamento che l'autore vuole tratteggiare necessita di veder mitigata la sua prorompente carica d'inquietudine psicologica in una declinazione più articolata delle singole forze che la compongono. Solo in tal modo il poeta può permettere alle tensioni ad essa soggiacenti di attenuarsi e di farsi meno dolorose. Magno, pertanto, decide di sviluppare i temi del sonetto I Io nell' arco di altre quattordici liriche. In esse si scorge la presenza di un duplice binario sul quale corrono, in direzioni opposte, le due parabole tematico-narrative che attraversano la sezione. Da un lato si assiste alla metamorfosi e alla purificazione dell' amore, cui il poeta, «seppure'lvigor in luipergli anni langue», non vorrebbe rinunciare; dall'altro si osserva una consapevole presa di coscienza dell' intrinseca pericolosità della natura del sentimento amoroso (seppur sublimato) e della conseguente necessità di doversene separare.

Questo doppio movimento appare articolarsi in tre fasi: nella prima (rime II2-IIG) vengono cantati $i$ «novi d'amor caldi sospiri», nella terza (I22-I24) le sofferenze che per sua stessa natura causa l'amore (da cui il poeta decide di allontanarsi definitivamente), mentre nella seconda (II7I2I) vengono riproposti in modo alterno i motivi sviluppati nelle altre due fasi, oggettivando nell'avvicendamento delle tematiche la precarietà della situazione del poeta in bilico tra le speranza offerte dal nuovo sentimento e la nuova consapevolez:a.

Il sonetto IIO, come accennato, funge da prologo e da riassunto delle tematiche della silloge, anticipandone gli sviluppi e collegandosi per mezzo di una esplicita immagine (v. 8, «m'è qual tra vaghi fior mortifer'angue») al sonetto I23, che chiude la sezione. In questa lirica (nella IIO), rideclinando il tema della canzone 32 di Della Casa, Arsi, et non pur la verde stagion fresca, il poeta si descrive come un nomo ormai non più giovane, che ha molto sofferto a causa della passione amorosa. Nei versi egli si rivolge direttamente al dio Amore ricordandogli come gli siano «mortiferi» quei «martiri» che in più «verde età» aveva accettato di sopportare, e lo prega di allontanarsi definitivamente da lui.

Se la richiesta del poeta venisse esaudita, la lirica potrebbe svolgere da sola la funzione di cerniera tra le rime amorose e quelle spirituali, 
presentando l'autore ormai ravveduto dall'antica passione e «ad altro fin rivolto». Ma la lirica successiva (la III, Già non usato ardor nel freddo petto) disattende questa aspettativa, presentando il poeta di nuovo in preda al sentimento amoroso. É interessante notare, però, che non è propriamente Eros a costringe il poeta a provare questo sentimento, ma l'influsso astronomico del pianeta Venere. ${ }^{48}$ Dietro questo particolare potrebbe celarsi semplicemente un gioco retorico ispirato alla variatio (il nome del pianeta, infatti, richiama la divinità omonima, madre di Cupido), ma il tentativo di attribuire la causa del nuovo sentimento all'influenza incontrastabile di una stella potrebbe anche apparire come una sorta di excusatio del nuovo stato di innamoramento, che risulterebbe allora una condizione imposta al poeta per necessità dall'influsso degli astri (e di cui egli, di conseguenza, non avrebbe colpa). Al contempo, il riferimento all'influenza di Venere potrebbe alludere anche ad un altra importante circostanza. Sebbene la descrizione dei nuovi sentimenti dell'autore ( $v v \mathrm{I}-4$ ) richiami la quinta stanza della canzone 32 di della Casa, ${ }^{49}$ confermando cosi l'ipotesi che sia Amore a richiamare il poeta tra le sue schiere riaccendendolo di "voglie e desiri», l'aggettivazione utilizzata nel sonetto allude ad un sentimento diverso da ogni altro che egli abbia mai provato. Magno parla, infatti, di «non usato ardor», di «cangiate voglie» e di «novi d'amor sospiri》. Siamo di fronte al sorgere di un affetto dai tratti finora sconosciuti, un «nobil amor» (v. I4), la cui comparsa, forse, anche per questo viene posta sotto il «benigno aspetto» della «bella Venere». E proprio in quest'ottica possono trovare spiegazione i riferimenti alla canzone dellacasiana dal momento che tramite quest'ultimi si sottolinea l'eccezionalità della condizione amorosa descritta: il poeta fiorentino, infatti, alludeva ad un suo improvviso ringiovanimento, Magno parla di un sentimento dai tratti nuovi e, come si cercherà di mostrare, casti ed eterei.

La novità dell'affetto e la sua purezza sono indicate anche nel sonetto II3, Vive in me 'l cor de la mia nobil diva, strettamente legato al precedente grazie alle didascalie anteposte ai due testi («Primo»e "Secondo») che si leggono nella stampa. Il nuovo sentimento riempie l'animo del poeta di «meraviglie nove», lo metamorfizza ( $v v$. 3-4, «in altr'uom mi trasforma e m'alia dove / aspirar il pensiero a pena ardiva») e lo innalza verso il ciel ( $v v$. 5 -6, " di morto ch'era in me l'ingegno avviva, / l'ale gli presta e verso il ciel le move»). 
Le tematiche e le immagini di questa lirica torneranno nella II6, O del mio nobil foco alta mercede, l'ultima del primo dei tre gruppi in cui si può dividere la sezione. Tra questo sonetto ed il iI6 se ne trovano altri due (II4 e IIS) in cui si celebra la bellezza degli occhi dell'amata. Nel componimento IIs, Da duo begli occhi al sol di luce eguali, l'amata è presentata in compagnia di «Bellezza» ed «Onestà», grazie alle quali vive sicura da ogni affetto impudico poiché, se la prima rende i cori degli innamorati troppo audaci, la seconda li spaventa. Si può leggere in questa caratteristica un'ulteriore dimostrazione della nuova natura (purificata) del sentimento amoroso che prova il poeta.

Nel sonetto II7, Ardo amante felice e del mio foco, è declinato un tema che appare fortemente antitetico rispetto a quanto è stato presentato finora nella silloge: quello della gelosia. La prima quartina presenta le nuove angosce del poeta e si conclude con una frase gnomica di tono universale: «il reo sospetto amaro / che là 've regna Amor mai sempre ba loco» ( $v v \cdot 3-4)$. Nella seconda stanza si descrivono $i$ sintomi provati dall'amante a causa della gelosia, mentre le terzine ribattono sul fatto che quest'ultima sia una componente statutaria della natura dell'amore (tanto più connaturata con esso tanto più l'oggetto del sentimento è un «tesoro raro e prezioso»). Secondo quanto affermato, il poeta, nonostante l'amata gli dimostri cieca fedeltà ( $\mathrm{v} v$. 9-I0, «e benché splenda a me pietate e fede / dal mio bel sole»), non potrà esimersi dall'essere geloso e dal soffrirne. Magno cerca in tal modo d dimostrare la pericolosità intrinseca del sentimento amoroso. Appare evidente che nei versi del sonetto l'autore, più che rivolgersi ad altri, sta cercando di convincere soprattutto sé stesso dell'assoluta necessità di allontanarsi da ogni affetto terreno (intrinsecamente nocivo per l'animo) al fine di poter tendere verso il Bene supremo. Questa lirica, dunque, può essere considerata come il primo di quei testi della sezione in cui si scorgono $i$ tentativi che il poeta compie per allontanarsi da un mondo i cui pericoli gli sono ben noti, ma che, al contempo, suscita in lui ancora profonde attrattive.

Nei componimenti successivi si manifesta questa situazione nell'alternanza con cui le varie liriche dipingono dei momenti di attaccamento all'amore ed altri in cui l'autore si fa cosciente delle minacce di tale sentimento. Il sonetto II 8 , O bench'ingiusto a me dolce lamento, è un ritorno alla sfera degli affetti. Nella fictio di rispondere ad una ingiusta lamentela dell'amata (che lo accusava di provare nei suoi 
confronti solo un «leve ardor»), il poeta trova il pretesto per cantarle tutto il suo amore. Segue un sonetto, il IIg, Austro s'ascondi in ciel del sol la luce, in cui, sebbene si descriva un semplice quadro tibulliano composto dalla preghiera rivolta dall' autore ai venti affinché facciano cessare la tempesta che lo tiene lontano dall' amata e gli concedano di raggiungerla, si pone significativamente l'accento su un altro aspetto statutario della natura d'amore che, come la gelosia, procura inevitabili sofferenze: la lontananza. La lirica successiva, la I20, Doppia bellezza in voi, doppio in me foco, costruendosi sul parallelismo tra la bellezza dell'aspetto e quella dell'anima della donna (che si rispecchiano e si accrescono a vicenda), tesse nuovamente le lodi dell'amata. L'ultimo sonetto di questa secondo movimento della sezione è il I2I, di cui si riporta il testo.

Quand'ergo al ciel le tante grazie ond'io ardo felice e la mia dea risplende, modesta ella il ver nega, e insieme rende maggior la sua bellezza e 'l foco mio.

Ch'ove pur del bel volto il tempo rio furi alcun pregio, Amor nulla s'offende, tant'altro ampio tesoro in lei comprende che fa beato a pien l'occhio e 'l desio.

E se sue doti eccelse e pellegrine si fan per gli anni a par di sé men belle, a par de l'altre son rare e divine.

Ché non ponno oscurar l'ore rubelle celeste lume: e benché 'l sol decline, vince un sol raggio suo tutte le stelle.

Anche questo componimento, come il II9, appare a prima vista solamente un elogio della donna, le cui grazie, quando il tempo della giovinezza sarà trascorso, saranno sempre degne di lode poiché, anche se appariranno «men belle» a paragone di come sono state, saranno sempre «rare e divine» rispetto a quelle altrui. Ma in sordina, accanto a questo tema elogiativo, si insinua nel sonetto un'altra immagine: quella della vecchiaia che inevitabilmente attende ogni essere umano. La meditazione sul destino ineludibile cui $i$ viventi si devono sottomettere (già declinata nella raccolta) si lega qui a quella sull'amore, alludendo cosi ad un altro degli aspetti della natura di questo sentimento che provocano sofferenza. 
La lirica si chiude con un'immagine icastica e doppiamente significativa: quella di un raggio di sole al tramonto. Da una parte essa anticipa le tonalità degli ultimi componimenti della sezione (I2I-I24), in cui l'autore si distaccherà definitivamente dagli affetti terreni per avvicinarsi, purificato, alle liriche spirituali; dall'altra rappresenta il commiato definitivo dalla sua amata. Il delicato paragone del verso I4 è l'ultima lode della raccolta che il poeta rivolge alla donna, che, come il sole, vince anche nel tramontare ogni altra stella.

Richiamandosi ai precedenti componimenti meditativi (IIT, III, I2I), nel sonetto I22, Se 'l tempo, o mio bel sol, ch'io v'ho presente, il poeta tesse un bilancio definitivo della sua esistenza. Essa appare composta da pochissimi attimi felici e da molte sofferenze,, ${ }^{\text {so }}$ legate ai periodi di lontananza dall'innamorata ( $v v$. 5-6, «felice a voi vicin, misero assente; / morto son senza voi, con voi son vivo», che richiamano il tema del sonetto II9), al desiderio disatteso di poter provare una gioia maggiore di quella concessagli (vv. 7-8, «ma il duol è un mar, la gioia un picciol rivo / che sete accresce a la mia voglia ardentes, che ricordano $i$ $v v$. 4-8 del componimento II7, dedicato alla gelosia $)^{5 \mathrm{I}}$ ed alla fortuna avara che invola ogni istante e che spinge veloce la vita umana verso il suo inevitabile fine (vv. 9-II, «Abi, che del mio tesor troppo m'invola / fortuna avara; e quest'umana vita / troppo senza spronarla al suo fin volas, che allude al sonetto I2I). Tutto cio lo spinge a rinunciare definitivamente agli affetti terreni, che sublimano nell'unione spirituale delle anime dei due amanti (vv. I2-I4, "Ma se al corpo è la via tronca e impedita, / fia l'alma a voi, sua luce amata e sola, / col desir sempre e col pensiero unita»).

Maper decretare il suo assoluto e definitivo abbandono del mondo (e, forse, per autoconvincersi di affrontare tale distacco), Magno sembra necessitare di uno stimolo ancora più forte. Concepisce allora una sorta di prova definitiva della mendacità di ogni sentimento terreno, che, ingannando $i$ sensi, allontana dal sommo Bene. La lirica I23, Qual pianger più debb'io? La rotta fede, presenta il tradimento da parte dell'amata della fede d'amore promessa al poeta. Il sonetto non assume $i$ tratti di una requisitoria o di un'invettiva verso la donna, bensi quelli di un'apostrofe rivolta al furtivo amante con cui fugge, cui il poeta, saggio della sua molta esperienza, predice la stessa sorte ora toccata a lui. ${ }^{52}$ L'autore non appare nemmeno sorpreso dalla situazione, che sembra venir accettata coscientemente come la dimostrazione della fondatezza 
dei sospetti che la gelosia gli aveva suscitato in precedenza. Il tradimento documenta inconfutabilmente la pericolosità di ogni affetto terreno, anche del più sublime (come ormai si presentava il sentimento verso l'amata) e spinge di conseguenza a fuggire l' amore ed a rinunciare per sempre ad esso. A riprova della natura definitiva della risoluzione adottata e, di conseguenza, della raggiunta conclusione del percorso intrapreso in questa sezione, ritorna nel verso 6 del sonetto ( $«$ senza timor d'occulto serpe andai») l'immagine del verso 8 del IIo («m'è qual tra vaghi fior mortifer'angue»), con cui si apriva la silloge. Compreso (ed ora accettato) che l'amore è un «mortifer'angue», il poeta può pentirsi e distanziarsene, rivolgendo il pensiero alla meditazione religiosa.

Prima dell'inizio della sezione delle rime spirituali, però, viene interposto un altro sonetto, il I24, strettamente collegato con il precedente dalla didascalia a testo «Secondo» (mentre il I23 veniva indicato come « Primo»).

Errai, m'accuso: falso, empio sospetto fe' torto al ver; pentito, il cor sen' dole. nero il bianco stimai, tenebre il sole, e pien di frode amor puro e perfetto.

Trafisser l'innocente e nobil petto le scelerate mie stolte parole; e 'l bel volto che vita a me dar suole bagnò di pianto giusta ira e dispetto.

O gelosia, d'Amor perversa figlia! $\mathrm{O}$ di dolce radice amara pianta! Misero chi a le tue vane ombre s'appiglia.

Ma tu prego, mia dea, perdona tanta colpa e serena le turbate ciglia: ch'ov'è più error, più la pietà si vanta.

In questa lirica compare una commovente palinodia delle accuse rivolte alla donna amata. Non ritengo che quest'ultimo componimento sia da leggere come una ritrattazione delle argomentazioni che hanno spinto l'autore ad abbandonare gli affetti, né una prova della loro insincerità (anzi la conversione delpoeta appare onestamente autentica), mapotrebbe, invece, essere stato scritto in nome di quel puro affetto che almeno un tempo (se alla fine della raccolta non è più praticabile) l'ha legato all'amata. Magno sa di essere pronto a rivolgere altrove il suo animo, ma non vuole chiudere la parte del suo libro di rime di argomento 
terreno con un'immagine che offenda la memoria della sua donna, soprattutto se quell'immagine (come si può supporre) non registra una reale esperienza biografica, ma risulta funzionale al poeta (strutturalmente e psicologicamente), fungendo da controprova della pericolosità del sentimento amoroso e, dunque, spingendolo ad un definitivo allontanamento da esso. Nel sonetto l'autore non allude nemmeno alla possibilità di una rinascita della passione (circostanza che dimostra l'autentico distaccamento di Magno dal mondo), accusa solo (retoricamente) la gelosia di averlo tratto in inganno e chiede perdono all'amata, prima di darle l'ultimo addio.

Giacomo Comiati 
I. L'edizione cui si fa riferimento è Rime di Celio Magno et Orsatto Giustinian, Venezia, Muschio, I600. Il presente studio si basa su quest'ultima sia per quanto riguarda le citazioni, sia per i riferimenti all'ordine con cui i componimenti sono disposti. L'analisi è condotta unicamente sulle I 37 rime che compongono il canzoniere, tralasciando, invece, quelle di corrispondenza stampate in calce all'edizione. Il Canzoniere del Magno si può leggere anche nella versione approntata da Francesco Erspamer per l'ATL. Per le notizie sull'attività lirica e sulla tradizione del testo cfr. F. Erspamer, Per un'edizione critica delle rime di Celio Magno, in «Studi di filologia italiana», 4I, I983, pp. 45-72, e ID., Lo scrittoio di Celio Magno, in Il libro di poesia dal copista al tipografo, a cura di M. Santagata e A. Quondam, Modena, Panini, I989, pp. 243-50.

2. Il primo sonetto a stampa di Celio Magno apparve nella raccolta Del tempio alla divina signora Giovanna d'Aragona (Venezia, Pietrasanta, I 55 ). In quegli anni numerose sue composizioni furono accolte in diverse miscellanee oppure inserite come rime di corrispondenza in canzonieri di contemporanei (cfr. E. A. CicoGNA, Delle iscrizioni veneziane, vol. v, Venezia, Giuseppe Molinari stampatore, I 842, pp. 240-54). Una silloge più corposa di componimenti (circa una trentina) apparve nel volume De le rime di diversi nobili poeti toscani, curato da Dionigi Atanagi (Venezia, Avanzo, I 565). Anche La bella e dotta canzone sopra la vittoria dell'Armata della Santissima Lega, nuovamente seguita contro la Turchessa, scritto per la vittoria di Lepanto, ebbe una circolazione precedente alla stampa del i600, anzi, fu probabilmente l'opera più fortunata di Magno: infatti tra il i 57 I e il i 572 fu stampata sia autonomamente sia inclusa in numerose raccolte celebrative dell'evento. Sullo stesso tema Magno scrisse anche un Trionfo di Cristo per la vittoria contra' Turchi, che fu rappresentato il 26 dicembre I 57 I davanti al doge Alvise Mocenigo. Nel I 597 pubblicò inoltre la canzone spirituale Deus (Venezia, Farri) con un corposo paratesto di discorsi e interpretazioni ad opera di Ottavio Menini, Valerio Marcellini e Teodoro Angelucci.

3. Per alcune considerazioni sulla successione delle varie sezioni nel canzoniere cfr. G. Stella Galbiati, Epilogo sacro e libro: alcune considerazioni sulle Rime di Celio Magno, in Autorità, modelli e antimodelli nella cultura artistica e letteraria tra Riforma e Controriforma. Atti del seminario internazionale di studi, Urbino Sassocorvaro, 9-I I novembre 2006, a cura di A. Corsaro, H. Hendrix, P. Procaccioli, Roma, Vecchiarelli, 2007, pp. 369-85, in particolare pp. 369-71.

4. C. Galimberti, Celio Magno e il petrarchismo veneto, in Crisi e rinnovamento nell'autunno del Rinascimento a Venezia, a cura di V. Branca e C. Ossola, Firenze, Olschki, I991, pp. 359-72, p. 364.

5. C. Galimberti, Disegno petrarchesco e tradizione sapienziale in Celio Magno, in Petrarca, Venezia e il Veneto, a cura di G. Padoan, Firenze, Olschki, i976, pp. 31 5-32, p. 3 I 8.

6. Cfr. Galimberti, Disegno petrarchesco cit., in particolare pp. 3 I 7-i 8 . Per la sezione delle rime spirituali ed in particolare per la canzone Deus cfr. G. Stella Galbiati, Contributo per Celio Magno: una lettura della canzone Deus, insieme ai suoi antichi commentatori, in Studi di onomastica e letteratura offerti a Bruno Porcelli, a cura di D. De Cavilli, Pisa-Roma, Gruppo Editoriale Internazionale, 2006, pp. I 29-44, ed EAD., Epilogo sacro e libro cit., pp. 369-85. 
7. Galimberti, Disegno petrarchesco cit., p. 323.

8. Galimberti, Celio Magno e il petrarchismo veneto cit., p. 364. Nelle rime del poeta veneziano (Stella Galbiati, Epilogo sacro e libro cit., p. 369) ha, però, individuato un altro Leitmotiv, le cui tracce sono molto più visibili all'interno della raccolta e «rispetto al quale la sezione sacra acquista organicità e funzionalità». Questo fil rouge è il tema della morte. La raccolta si apre, infatti, con «l'immagine del canzoniere-sepolcro»; il mondo intero è descritto come uno spettacolo funebre e l'eroismo e la virtù non sono altro che illusioni che si dissolvono di fronte alla rivelazione ultraterrena.

9. Per la centralità del tema del locus amoenus nella lirica di Magno mi si conceda di rimandare ad un mio articolo: G. Comiati, Presenze oraziane nelle rime di Celio Magno, in Canzonieri in Transito, a cura di A. Metlica e F. Tomasi, Milano, Mimesis (in corso di stampa).

ı. L'«augellin», protagonista della canzone 43, divine il simbolo dell'ambiente idillico in cui è ritratto e, per sineddoche, il suo principale rappresentante. L'animale si fa allora oggettivazione della rinata vitalità di cui la lirica è testimone, tramite la quale la poesia può rigenerarsi.

I I. Come nel canzoniere dellacasiano il breve ciclo di canzoni 45-47 fa da spartiacque tra le liriche di tono prettamente amoroso che lo precedono e quelle di meditazione morale che seguono, così le due canzoni 42 e 43 segnano un "prima" e un "dopo" nell'articolazione dei componimenti di Magno (sebbene in quest'ultimo i temi trattati nelle due sezioni siano invertiti rispetto a Della Casa: nel liber del veneziano, infatti, si sono approfondite fino allo "spartiacque" le tematiche morali, mentre solo in seguito si sviluppa la lirica d'amore).

I 2. Si fa riferimento a $R v f 43$, Vago augelletto che cantando vai, e a due sonetti delle Rime bembiane, il 48, Solingo angello, se piangendo vai, e il 56, O rossigniuol, che 'n queste verdi fronde. Come aveva già notato Antonio Pilot, però, in questi ultimi due casi «di simile [con la canzone del Magno] non v'è che l'idea della maggior felicità dell'uccello piuttostoché nel poeta» (A. РіLот, Le canz̧oni di Celio Magno in relaz̧ione colla lirica veneta del tempo, (parte i e II) in «Ateneo Veneto», xxxir, sett.-ott. i 909 e nov.-dic. I 909, pp. I 17-308, in particolare p. 149).

i 3. Cfr. Pilot, Le canzoni di Celio Magno cit., pp. i48-49; Galimberti, Celio Magno e il petrarchismo veneto cit., p. 370; ed E. TADDEO, Leopardi lettore di Celio Magno, in Letteratura e Critica. Studi in onore di Natalino Sapegno, a cura di W. Binni, A. Castellani, P. Chiarini, M. Colesanti, A. Lombardo, G. Macchia, G. Melchiori, M. Praz e C. Salinari, vol. I, Roma, Bulzoni, I974, pp. $635-47$, in particolare p. 638.

14. Giovanni Della Casa, Rime 63, 9-I 4.

I s. Il giovane Esaco fu preso d'amore per la ninfa Esperie. Costei, mentre lo fuggiva, fu morsa da un serpente e morì. L'amante, disperato, si buttò in mare da una rupe, ma Teti, impietosita, lo trasformò in cormorano. Cfr. Ov. Met. xI, 749-95. 
i6. G. Tanturli (a cura di), Giovanni Della Casa, Rime, Milano-Parma, Fondazione Bembo-Guanda, 2001, p. I 87.

i 7. Galimberti, Celio Magno e il petrarchismo veneto cit., p. 37 I.

i 8. L'immagine deriva da LatTanzio (Instit. div. i, i i: «Instituit pompam, in qua Jupiter cum ceteris diis ante currum triumphantis ducitur catenatus») anche se mediata dall'esempio petrarchesco (TC. I, I60).

I9. Per curare le sofferenze amorose Cicerone (Tusc. iv, 35, 74) invitava a mutare luogo, ma la denuncia dell'inefficacia del rimedio è già in ProperzIo (II, 3O, I-2, 7), prima che nei moderni (cfr. a titolo esemplificativo Rvf 35, I 2-I4; Ariosto, Orlando Furioso 28, 89,8; oppure BЕмво, Rime 69, 4).

20. Altri esempi della consonanza tra Della Casa e Magno sono forniti, ad esempio, dalla prossimità del sonetto 49 del veneziano, Questa selvaggia mia cruda guerriera, con il sonetto is del fiorentino, Quella che del mio mal cura non prende. Nel primo la donna amata è dipinta come «cruda guerriera», che spinge il poeta in fin di vita, ma sta attenta «perch'ei non pera» (v. 4), altrimenti finirebbe così il suo «impero» su di lui. Quest'immagine di Magno è un'eco di Della Casa: «non gradisce 'l mio cor, e no 'l mi rende, / perch'ei sempre di lacrime trabocchi; / né vol ch'i' pera, e perché già mi tocchi / morte col braccio, ancor non mi difende» ( $\left.5,4^{-8}\right)$. Ugualmente sono molto affini il sonetto 50 di Magno e la canzone 45 del fiorentino. Il primo declina il tema delle sofferenze della donna crudele, per le quali egli prova grande pena (quasi fossero le sue), ma contemporaneamente (e, forse, involontariamente, come se fosse frutto di un egoismo primitivo che vive in lui separatamente dai sentimenti affettivi) le interpreta come giusta punizione di quanto l'amata gli ha fatto patire («Né ciò men, lasso, a me tormento adduce; / anzi sì grave e rio l'alma il sostiene, / ch'io scorgo presso il fin de la mia luce. // Sol un conforto in vita il cor mantiene: / che mentre il duol madonna a tal conduce, / vendetta fa de le mie lunghe pene», 9-I 4). Similmente Della Casa: «sì ch'ella caggia sanguinosa e pèra, / e quel selvaggio core / ne le sue piaghe senta il mio dolore; / e biasmando l'altrui cruda e guerrera / voglia, il suo proprio errore / e la sua crudeltà colpi e condanni: / e fia vendetta de' miei gravi affanni / veder ne' lacci di salute in forse / l'acerba fera, che mi punse e morse» (97-105). Il particolare rapporto di vicinanza tra $\mathrm{i}$ due poeti è sottolineato anche da R. SCRIVANo in Il manierismo nella letteratura del Cinquecento, Padova, Liviana Editrice, I959, in particolare cfr. pp. 99-108.

\section{2i. G. Tanturli in Giovanni Delda Casa, Rime cit., p. 49.}

22. Un'analisi più accurata della funzione e dell'utilizzo del metro della canzone all'interno del canzoniere di Magno è ancora in corso ad opera di chi scrive. Si rimanda ad altra sede lo studio più approfondito dei risultati che potrà fornire questa indagine. Per un'analisi contenutistica delle canzoni di Magno cfr. РiLOT, Le canzoni di Celio Magno cit.

23. Il tema della funzione eternatrice del canto poetico è declinato nel proemio del canzoniere, come anche in altri componimenti (ad esempio, sebbene in tono allusivo, nel sonetto 7, Dentro al vaso mortal giacendo sotto). Per il tema si rimanda a Comiati, Presenze oraziane cit. 
24. A differenza di quanto accade nella stessa edizione Muschio per le liriche per li capiversi di Giustinian, segnalate tramite l'utilizzo di lettere maiuscole per tutte le parole con cui iniziano i versi dei componimenti, nelle rime di Magno non viene impiegato questo espediente. Si può scoprire che il sonetto è acrostico solo grazie alla didascalia posta nella «Tavola delle Rime», escamotage con cui Magno non inserisce apertis verbis il nome dell'amata nel canzoniere, ma che, al contempo, non impedisce a «Paulina» di ricevere la lode semiesplicita che il suo poeta voleva tributarle. Cfr. G. Comiati, Componente paratestuale e didascalie nelle Rime di Celio Magno, in Questioni filologiche: la critica testuale attraverso $i$ secoli, a cura di J. L. Bertolio et alii, Firenze, Franco Cesasti, 2014 (in corso di stampa).

25. Son. 59: «Scrivi - mi disse Amor, - ch'al mondo vive / sotto velo mortal celeste dea / che con un guardo sol l'anime bea, / d'ogni altro ben fuor del bel volto schive. // Dì che fiamme ho per lei sì calde e vive / che di ghiaccio eran l'altre ond'io v'ardea; / dì che 'l pensier formar non basta idea / ch'a l'alto segno de' suoi pregi arrive. // E 'l nome puoi tacer: ch'a tanta luce / scerner da l'altre alcun non dubbia od erra; / né per farlo palese il sol si mostra. // Ma goda quanto può la vita vostra / del suo splendor: ché raro il ciel produce / di tal beltà le meraviglie in terra».

26. Si pensi ai famosi dipinti di Veneri allo specchio di Bellini (ad esempio «Giovane donna nuda allo specchio», I 5 I 5 , Vienna, Kunsthistorisches Museum) o di Tiziano (ad esempio, «Venere allo specchio», I 555 , Washington, National Gallery of Art; quest'ultimo è un riferimento più appropriato perché nei quadri appare anche la figura dell'amorino che regge lo specchio). Con questi esempi legati alle arti figurative la lirica sembra intrecciare un gioco di rimandi sulla base dello stesso principio dei riferimenti tra ritratti e poesie dedicate al ritratto (cfr. L. Bolzoni, Poesia e ritratto nel rinascimento, Roma-Bari, Laterza, 2008). Per il tema del rapporto tra Magno ed alcuni esponenti dell'ambiente artistico veneziano (in particolare Domenico Tintoretto) cfr. B. Mazza Boccazzi, Ut pictura poesis: Domenico Tintoretto per Celio Magno, in «Venezia Cinquecento. Studi storici dell'arte e della cultura», XI, n. 22, 200I, pp. I67-75.

27. Lo schema delle terzine CDE ECD ricorre solo altre due volte nella raccolta di Magno: nei sonetti i 7 e 8 I.

28. Il motivo è di chiara matrice oraziana (cfr. Hor. Carm. I, I I; Epod. I 3, 3; Epist. I, I I, 23), ma si intreccia con una componente properziana: nell'elegia I, I9 (25-26), infatti, il poeta umbro declina la tematica del carpe diem rivolgendo l'invito del venusino espressamente a sé stesso e all'amata ( Quare, dum licet, inter nos laetemur amantes: / non satis est ullo tempore longus amor»), fornendo così un precedente per i versi di Magno.

29. Nel canzoniere di Magno si possono ritrovare molti luoghi più o meno legati esplicitamente alla poesia di Properzio. Oltre a questo caso, si considerino, a titolo esemplificativo, il sonetto 65 , che tratta il topos del paraklausithyron (cfr. Prop. I, 6), o alcuni luoghi della canzone 8 (in particolare il sottotesto dell'invettiva contro l'avarizia lì declinata è PROP. III, 7) o della canzone 70 (cfr. in particolare l'immagine del poeta come magister amoris presente nei vv. 42-48, riconducibile a Prop. I, I), come anche molte immagini declinate in alcuni sonetti del ciclo 
amoroso (l'immagine della donna come «cruda guerriera» son. 48 e 49 è già in Prop. II, 30, I-2; così come il tema e le immagini del sonetto 62 possono essere stati ispirati da PRop. I, 8, I-I 2). Il peso dell'influenza properziana non è per nulla irrilevante per le raccolte poetiche cinquecentesche (cfr. A. La Penna, L'integrazione difficile. Un profilo di Properzio, Torino, Einaudi, 1977, pp. 250-299, e E. Villa, La «musa tenuis» di Properzio nella poesia de Cinquencento, in Properzio nela letteratura italiana. Atti del Convegno Nazionale (Assisi, I 5-17 novembre 1985), a cura di S. Pasquazi, Roma, Bulzoni, I987, pp. 93-I I 5). Ed il caso di Magno, una volta studiato opportunamente, rappresenterà un'importante capitolo della storia dell'influenza dell'elegiaco latino nel panorama rinascimentale.

30. Un'ulteriore testimonianza della calibrata architettura a specchio su cui si compone la sezione è offerta dalla declinazione in alcuni versi della canzone 69 delle stesse immagini presenti nei componimenti che aprivano la silloge in lode della donna: cfr. 57, 3-4 «soavi fiamme in cui more e rinasce / per miracol d'amor nova fenice» e 69, 59-6r «e ch'ivi, ardendo, moia; / ben ch'indi ognor rinasco, / quasi fenice nova».

3r. Le informazioni biografiche sull'autore che si possono consultare attualmente non specificano l'anno in cui Magno si trasferì in Spagna, ma in attesa di una ricerca d'archivio che chiarifichi la situazione, si può almeno fissare come termine post quem per la sua partenza il i 575, dal momento che il poeta seguì Vendramin nella sua ambasceria come segretario del Senato e che questa carica gli venne affidata solo a partire da quell'anno. Cfr. D. Ghirlanda, Celio Magno (ad vocem), in $D B I$, vol. 67, pp. 496-98.

\section{Pilot, Le canzoni di Celio Magno cit., p. 267.}

33. Si ricorda, com'è noto, che Petrarca decide di collocare il sonetto 92, in morte di Cino da Pistoia, tra rime di argomento amoroso (Rvf 9o, Erano $i$ capei d'oro a l'aura sparsi e Rvf 93, Più volte Amor m'avea già detto: scrivi). Per quel che riguarda il precedente dellacasiano, invece, si può osservare che il ciclo di sonetti I 2-I 3 , in morte di Marcantonio Soranzo, si interpone tra i sonetti dedicati alla gelosia (6-8) e quelli in cui si declina il tema delle sofferenze amorose (I4-I6). Anche la classicità forniva un precedente simile: Ovidio negli Amores collocava tra elegie amorose un componimento in morte di Tibullo (III, 9). Si può inoltre plausibilmente ritenere che Magno guardi all'esempio offertogli dal Della Casa nel momento in cui decide di utilizzare quasi nella metà esatta della sua raccolta il metro della canzone, in modo che essa funga da perno per l'intero canzoniere, esattamente come fa il fiorentino con la canzone Arsi; et non pur la verde stagion fresca (la numero 32 delle 64 liriche che compongono il suo libro di rime).

34. Si può immaginare che Molino venga celebrato da Magno come suo maestro poetico, leggendo la terza strofa della canzone. Magno elogia l'amico come cantore «del gran Re celeste», della patria, del vizio e della virtù e lo definisce capace di insegnare con le sue rime d'amore «a quanto mal s'apprende / chi 'l senso in guida prende / e che mortal beltà tanto s'apprezza / quanto ella è scala all'immortal bellezza» (70, 45-48). In questi versi si legge, ovviamente, un elogio della poesia di Molino, ma, al contempo, essendo tanto affini le intonazioni poetiche dei due autori, si potrebbe intravvedere in essi un catalogo della 
tematiche sviluppate da Magno stesso (già PiLot, Le canzoni di Celio Magno cit., p. 268 scriveva: «nella strofa terza il poeta cerca ritrarre i pregi della Musa del Molin con parole ch'io, più volentieri, adatterei a quella del Magno stesso»). Il terzo ed il quarto tema dell'elenco (quelli morali) all'altezza della canzone 70 sono già stati trattati nel canzoniere di Magno (cfr. i primi 26 componimenti), i primi due (le rime spirituali e quelle in lode della patria), invece, verranno declinati nel prosieguo della raccolta, mentre il tema amoroso (il quinto dell'elenco) è trattato nelle liriche appartenenti alle sezioni collocate prima e dopo questa canzone. Il breve epitrocasmo, allora, si potrebbe leggere come compendio delle tematiche già incontrate nel liber di Magno ed anticipazione programmatica di quelle della seconda metà della raccolta. La canzone, in tal modo, si doterebbe di un plusvalore strutturale e strutturante all'interno del canzoniere.

35. La tematica del tradimento dell'amata da parte del poeta (che si invaghisce di un'altra donna) è declinata in $R v f 27 \mathrm{I}$. Si noti che il sonetto petrarchesco richiama lo stesso motivo sviluppato nella Vita nova (cfr. M. SAntagata, I frammenti dell'anima, Bologna, Il Mulino, 2004² (1992), pp. 198-200).

36. La didascalia alla canzone 86 recita: «Trovandosi alla corte di Spagna l'anno I 576 , secretario (sic.) con l'illustrissimo signor Alberto Badoaro, cavalier allora ambasciatore presso sua Maestà cattolica, e sorpreso da una ferma imaginazione di dover morire in quelle parti, compose questa canzone».

37. Il nome Filli ritorna diverse volte già nelle Bucoliche virgiliane (cfr. Ecl. 5, io; 7 , I 4; 7, 59; го, 36) prima ancora che nell'Arcadia di Sannazaro o nell' Aminta tassiano. Ma forse il precedente più significativo è Orazio, Carm. IV, I I, che definisce Filli «meorum finis amorum (non enim posthac alia calebo femina)» (3 I34). Cfr. a questo proposito E. Oliensis, Erotics and gender, in The Cambridge companion to Horace, a cura di S. Harrison, Cambridge, Cambridge University Press, 2007, pp. $22 \mathrm{I}-34$, in particolare p. 232.

38. Il primo verso del sonetto 89 si richiama agli incipit della prima e della settima egloga virgiliana (Ecl. I, I «patulae recubans sub tegmine fagi» e 7, I «forte sub arguta consederat ilice Daphnis»).

39. Alcuni esempi dell'immagine del frutto donato alla donna, cui soggiace un simbolismo erotico, si trovano in Verg. Ecl. 3, 70-7 I; Prop. I, 3, 24; Ov. Met. x, 664 e sgg.

40. Si riporta qui la prima quartina del componimento che restituisce l'atmosfera della lirica (T. TAsso, Rime, 367 ), molto simile nei toni a quella di Magno: «Aminta, poi ch'a Filli non dispiacque / del medesmo desir mostrarsi accesa, / e ch'ella a questo sol già tanto attesa / ne le sue braccia alfin nuda si giacque».

4I. L’ultimo (il 98) è, però, metricamente una ballata.

42. M. Manfredi, Cento madrigali, Mantova, appresso F. Osanna, i 587 , p. i i 5.

43. Il madrigale di argomento pastorale è usato anche da Tasso. 
44. Il paragone floreale presente nel componimento 95, Benché ad aprir con lei la bella aurora, come anche l'utilizzo di una strofa di tutti settenari, richiama la canzonetta alla viola di Chiabrera. Non è ovviamente certa alcuna tangenza certa con il poeta ligure, ma si coglie almeno in questo e nei successivi esempi la vitalità di un clima di costante ed ininterrotto sperimentalismo lirico che a Venezia si era prodotto sin dagli anni quaranta del Cinquecento. La gara canora tra un pastore ed un «augelletto» descritta nel madrigale 96, Mentre in verde boschetto, potrebbe alludere ad un sonetto di Giacomo Zane, Piangea cantando, concepito come gara fra un uccellino e l'eco. Il componimento 97, Di Medea cruda è quella, che la didascalia nella «Tavola delle Rime» ci dice essere «tradotto dal greco antico», appare la resa poetica di un componimento dell'Antologia planudea, nello specifico

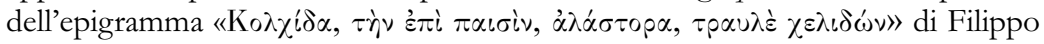
(I sec. d.C.), che si legge alla c. I $87 \mathrm{v}$ dell'Antologia di epigrammi greci stampata da Manuzio nel i 550 (Anthologia diaphorōn epigrammatōn archaiois [...], Venezia, Manuzio, I 550), oggi in Antologia di Planude, I6, I4I.

45. Pilot, Le canzoni di Celio Magno cit., p. 278.

46. Nell'edizione Muschio il titolo di «Spirituali» compare prima del sonetto i 25 , $O$ vago tanto e lusinghiero in vista.

47. Cfr. Galimberti, Celio Magno e il petrarchismo cit., p. 364.

48. Come si deduce dal testo e come sottolinea il poeta, a scanso di equivoci, nella didascalia che accompagna il sonetto nella «Tavola delle Rime»: «Dominava secondo gli astrologi in quell'anno della sua vita il pianeta Venere, calcolando dal giorno della sua natività, che fu alli $\mathrm{I} 2$ di maggio i $536 \%$. Per un precedente poetico in cui è descritto l'influsso della stella Venere cfr. Hor. Carm. II, I 9.

49. Cfr. i versi I-4 del son. i I del MAGNo («Già non usato ardor nel freddo petto / sento, e cangiate in me voglie e desiri; / già fra novi d'amor caldi sospiri / mi trovo in dolce e degno laccio stretto») con Giovanni Della Casa, Rime 32, 45-55 «Rendimi il vigor mio, che gli anni avari / tosto m'han tolto, e quella antica forza / che mi fea pronto, e questi capei tingi / nel color primo, che di fuor la scorza / come vinto è quel dentro non dichiari; / e atto a guerra far mi forma e fingi, / e poi tra le tue schiere mi sospingi, / ch'io no 'l recuso, e 'l non poter m'è duolo. / Or nel tuo forte stuolo / che face più guerrer debile e veglio? / Libero farmi il tuo fôra e 'l mio meglio».

50. Cfr. Giovanni Della Casa, Rime i 9 (in particolare, per quest'immagine, il v. 2, «molti anni tristi e poche ore serene»).

5 I. Son. i I 7, 4-8: «L'infinito mio ben mi sembra poco, / e provo notte in mezzo 'l dì più chiaro, / via più ricco d'ogni altro e via più avaro, / tra soave d'Amor penoso gioco».

52. Sul tema del poeta tradito che ricorda al nuovo amante della sua donna che in futuro toccherà anche a lui la sua stessa sorte cfr. Hor. Carm. I, 5 ; Epod. I 5 ; Prop. II, $25,2 \mathrm{I}-22$. 